\title{
Tissue dose estimation after extravasation of ${ }^{177} \mathrm{Lu}-$ DOTATATE
}

Perrine Tylski ( $\nabla$ perrine.tylski@chu-lyon.fr)

Hospices Civils de Lyon https://orcid.org/0000-0001-8974-6930

Géraldine Pina-Jomir

Hospices Civils de Lyon

Claire Bournaud-Salinas

Hospices Civils de Lyon

Patrice Jalade

Hospices Civils de Lyon

Original research

Keywords: Extravasation, dosimetry, 177Lu-DOTATATE

Posted Date: August 18th, 2020

DOI: https://doi.org/10.21203/rs.3.rs-57991/v1

License: (c) (i) This work is licensed under a Creative Commons Attribution 4.0 International License.

Read Full License

Version of Record: A version of this preprint was published at EJNMMI Physics on March 31st, 2021. See the published version at https://doi.org/10.1186/s40658-021-00378-3. 


\section{Abstract}

Background. Extravasation of radiopharmaceuticals used for vectorized internal radiotherapy can lead to severe tissue damage (1). Clinical management of these extravasations requires the preliminary estimation of the dose distribution in the extravasation area. Data are scarce regarding the dose estimation in the literature. This work presents a methodology for estimating the dose distribution after an extravasation occurred in September 2017, in the arm of a patient during a $7.4 \mathrm{GBq}$ infusion of Lutathera ${ }^{\circledR}(A A A)$.

Methods. A local quantification procedure initially developed for renal dosimetry was used. A calibration factor was determined and verified by phantom study. Extravasation volume of interest and its variation in time were determined using 4 whole body (WB) planar acquisitions performed at $2 \mathrm{~h}\left(\mathrm{~T}_{2 \mathrm{~h}}\right), 5 \mathrm{~h}\left(\mathrm{~T}_{5 \mathrm{~h}}\right)$, 20h $\left(T_{20 h}\right)$ and $26 \mathrm{~h}\left(\mathrm{~T}_{26 \mathrm{~h}}\right)$ after the beginning of the infusion and three SPECT/CT thoracic acquisitions at $T_{5 h}, T_{20 h}$ and $T_{26 h}$. For better estimation of initial extravasation volume, 3 volumes were defined on SPECT images using a 3D activity threshold. Cumulated activities and associated absorbed doses $\left(D_{1}\right.$, $D_{2}, D_{3}$ ) were calculated in the 3 volumes using the MIRD formalism.

Results. Volumes estimated using 3D threshold were $\mathrm{V}_{1}=1000 \mathrm{~mL}, \mathrm{~V}_{2}=400 \mathrm{~mL}$ and $\mathrm{V}_{3}=180 \mathrm{~mL}$. Cumulated activities were evaluated using a mono exponential fit on activities calculated on SPECT images. Estimated local absorbed doses in $V_{1}, V_{2}$ and $V_{3}$ were $D_{1}=2.8 \mathrm{~Gy}, D_{2}=5.4 \mathrm{~Gy}$ and $\mathrm{D}_{3}=7.8 \mathrm{~Gy}$. Evolution in time of local activity in the extravasation area was consistent with an effective local period (Teff) of 3 hours.

Conclusions. Rapid local doses estimation was permitted thank to knowledge of the calibration factor determined previous to accidental extravasation. Luthatera ${ }^{\circledR}$ lymphatic drainage was quick in the arm (Teff $=3 \mathrm{~h}$ ). Estimated doses were in the lower range of deterministic effects and far under soft tissue necrosis threshold. Thus no surgical rinse was proposed. The patient did not show any clinical consequence of theses extravasation.

\section{Background}

[ ${ }^{177}$ Lu-DOTA,Tyr(3)]octreotate ( ${ }^{177}$ Lu-DOTATATE) is an effective treatment of advanced well differentiated gastro-entero-pancreatic neuro-endocrine tumors (GEP NET) (2). It relies on the combination of ${ }^{177} \mathrm{Lu}$, a medium energy beta - emitter with a peptide, binding specifically to tumors. ${ }^{177}$ Lu emits beta radiation with a maximum energy of $498 \mathrm{keV}(79 \%)$, photonic radiations ( $\mathrm{Y})$ of $113 \mathrm{keV}(6 \%)$ and $208 \mathrm{keV}(10 \%)$ and a half-life of 6.65 days (3). Its physical characteristics are appropriate for tumor treatment, gammacamera imaging and dosimetry calculation.

${ }^{177} \mathrm{Lu}$-DOTATATE is used in clinical routine in several countries for GEP-NET. It received US FDA approval in January 2018 and EMA approval in September 2017. It is administered intravenously during 10-30 
minutes (4). As any intravenous administration, extravasation may occur during ${ }^{177}$ Lu-DOTATATE injection. The retention of beta emitter in soft tissues can lead to very severe tissue damage (1).

Extravasation cases were not reported in the literature for ${ }^{177}$ Lu-DOTATATE, but two extravasation cases of ${ }^{177}$ Lu-PSMA-617 have been documented. One case was presented as a commented image of the patient 2, 20 and $40 \mathrm{~h}$ post injection (5) and the second is a brief case report with low details on dose calculation (6). Dosimetric evaluations are rare in extravasation cases (1). Dose calculation requires several acquisitions, whole body or SPECT/CT, to determine an effective period.

In September 2017, during an intravenous administration of a first cycle of Lutathera ${ }^{\circledR}$ (Advanced Accelerator Applications) treatment in our center, extravasation occurred in the arm of the patient. The radioactive solution accumulated in the upper part of his arm. SPECT/CT and whole body images were acquired and dose was estimated.

\section{Methods}

\subsection{Initial phantom study}

Activity estimation in the extravasation area of the patient images was realized using a local quantification protocol initially developed for renal dosimetry with ${ }^{177} \mathrm{Lu}$. Two phantom studies had been performed prior to the incident : one to set a calibration factor $\mathrm{F}$ in counts/MBq/s for ${ }^{177} \mathrm{Lu}$ and one to evaluate its accuracy on SPECT/CT images.

The calibration phantom is a body shape phantom (PTW Body phantom ' $\mathrm{B}$ ') with a cover containing 3 cylindrical inserts (PTW cover D with 3 cylinders), of which 2 are fillable and one is made of Polytetrafluoroethylene to simulate bone. The inner diameter of the cylinders was $4.6 \mathrm{~cm}$. Volumes of the fillable cylinders were determined by weighing and were respectively 305 and $321 \mathrm{~mL}$. The volume of the inserts were slightly larger, yet in the range of magnitude of normal kidney volume, $202 \mathrm{~mL}$ for men and $154 \mathrm{~mL}$ for women (7). The two cylinder inserts (Figure 1) were filled with ${ }^{177}$ Lu-DOTATATE and placed in the body shape phantom filled with water without activity.

The verification phantoms used (Figure 2) were a cylindrical phantom (PTW Head Phantom ' $\mathrm{H}$ ') and the body shape phantom previously described, alternatively changed from one acquisition to the other. $\mathrm{A}$ heart insert made of two non-axial cylinders was used (PTW Heart phantom ' $C$ '). Only the inner cylinder, with an inner diameter of $4.4 \mathrm{~cm}$ and a length of $10 \mathrm{~cm}$ (weighted volume $150 \mathrm{~mL}$ ) was filled with ${ }^{177} \mathrm{Lu}-$ DOTATATE. The outer cylinder was filled with water and placed in the cylinder or body phantom filled with water.

A reference activity concentration $(A C)$ of $1 \mathrm{MBq} / \mathrm{mL}$ was considered, corresponding to a value measured in a patient kidney $24 \mathrm{~h}$ after ${ }^{177}$ Lu-DOTATATE, as reported in the literature (8). Six acquisitions around 7 days apart were performed for each phantom, to cover a wide range of activity concentration (AC) around this reference value. All images were acquired on a Symbia T2® camera (Siemens Healthcare) with a 
5/8" crystal and a MELP collimator. Images were acquired and reconstructed according to the parameters detailed in Table 2 and 3, which were determined using joint EANM/MIRD recommendations for quantitative ${ }^{177}$ Lu SPECT (3). Images were corrected for attenuation using CT and for scatter using a double energy window method.

Activities were determined using a dose calibrator, calibrated for ${ }^{177}$ Lu (MEDI $405 \AA$, Veenstra). The total activities in the calibration and verification inserts and the corresponding activity concentrations are reported in Table 1.

\begin{tabular}{|lllllll|}
\hline Acquisition & \multicolumn{2}{l}{ Calibration - Cylinder 1 } & \multicolumn{2}{c|}{ Calibration - Cylinder 2 } & \multicolumn{2}{c|}{ Verification - Cylinder } \\
\cline { 2 - 7 } & $\mathrm{A}(\mathrm{MBq})$ & $\mathrm{AC}(\mathrm{MBq} / \mathrm{mL})$ & $\mathrm{A}(\mathrm{MBq})$ & $\mathrm{AC}(\mathrm{MBq} / \mathrm{mL})$ & $\mathrm{A}(\mathrm{MBq})$ & $\mathrm{AC}(\mathrm{MBq} / \mathrm{mL})$ \\
\hline 1 & 2275 & 7.51 & 1136 & 3.54 & 471 & 3.14 \\
\hline 3 & 1700 & 5.61 & 849 & 2.65 & 354 & 2.36 \\
\hline 4 & 808 & 2.67 & 403 & 1.26 & 167 & 1.12 \\
\hline 5 & 388 & 1.28 & 194 & 0.60 & 80 & 0.54 \\
\hline 6 & 186 & 0.61 & 93 & 0.29 & 39 & 0.26 \\
\hline
\end{tabular}

Table 1. Activities and activity concentrations in the cylinders used for calibration and verification

\begin{tabular}{|ll|}
\hline & SPECT acquisition \\
\hline Spectrometric window & Main: $208 \mathrm{keV}+/-7.5 \%$ \\
& Scatter: $180 \mathrm{keV}+/-6 \%$ \\
\hline Acquisition conditions & $128 \times 128$ matrix \\
& $2 \times 30$ projections of $30 \mathrm{~s}$ \\
& Auto-contour \\
\hline
\end{tabular}

Table 2. Acquisition parameters for SPECT acquisitions

\begin{tabular}{|ll|}
\hline Reconstruction Algorithm & Flash 3D $®$ \\
\hline Iterations & 10 \\
\hline Subsets & 10 \\
\hline Post-filtering & Gaussian filter with 4 mm FWHM \\
\hline
\end{tabular}


Table 3. Reconstruction parameters for SPECT images

Images were processed using Matlab ${ }^{\circledR}$ (Mathworks). The number of counts was calculated in a volume of interest ( $\mathrm{VOI}$ ) equal to the known volume of the cylinders on the calibration images. The number of counts was divided by the acquisition duration and plotted against the activity. The calibration factor $F$ in counts/s/MBq was determined using a linear regression on this plot.

In the verification step, we tried to simulate a patient image processing: VOI was manually determined slice by slice on the CT images of the phantom and reported on SPECT images. The number of counts in the VOI was calculated and converted into activity using F. The calculated activity was compared to the known activity.

\subsection{Patient}

Extravasation occurred during the first cycle of treatment in a 70 year-old male patient, previously operated for small intestine neuro endocrine tumors, with several metastatic lesions in the liver, bone lesions and one subclavicular lymph node, all expressing somatostatin receptors on 111 ln-pentreotide scintigraphy. Nephroprotective amino acids solution was perfused intravenously in the right arm from 10:40 without any incident. Lutathera was injected in the left median cubital vein at 11:40 $\left(T_{0}\right)$. The routine injection protocol was used, using a pump, pushing the saline solution in the vial. The ${ }^{177} \mathrm{Lu}$ DOTATATE was then pushed in a second manifold to the vein of the patient, by the pressure increase in the vial. The flow was set to $100 \mathrm{~mL} / \mathrm{h}$, and then increased to 200 and $300 \mathrm{~mL} / \mathrm{h}$. At the end of the perfusion, the nurse noticed a swelling just over the left elbow. The patient did not complain of any pain. The nurse immediately stopped the perfusion and informed the nuclear physician, who confirmed the extravasation. Medical physicist and local referee for nuclear and radiological incident were informed.

The residual activity of Lutathera in the vial was measured using a dose calibrator, calibrated for ${ }^{177} \mathrm{Lu}$ (MEDI 405®, Veenstra).

Osmogel ${ }^{\circledR}$ dressings were placed on the injection site. The patient underwent several whole body and SPECT/CT images. Adapted interventions were used to stimulate the lymphatic elimination during several hours after the detection of the extravasation, including in the night: warming and elevation of the left arm and repeated self-massages.

\subsection{Imaging}

Whole body images were acquired with a spectrometric window of $208 \mathrm{keV}+/-10 \%$ and a $20 \mathrm{~cm} / \mathrm{min}$ speed. The first whole body image was acquired 2 hours $\left(T_{2 h}\right)$ after the beginning of the perfusion and 1 hour after the extravasation was detected. Following routine acquisition protocol, the patient urinated before this first acquisition.

A second whole body image was acquired 5 hours $\left(T_{5 h}\right)$ after the beginning of the perfusion. This WB images were followed by a SPECT/CT acquisition on the arm. All SPECT/CT images were acquired with 
the parameters provided in Table 2 and reconstructed using the parameters specified in Table 3. Scatter and CT-based attenuation corrections were applied.

The first two whole body acquisitions and the SPECT/CT images were used to make a preliminary estimation of the dose to the arm, a necessary step for deciding whether surgical rinse might be performed to avoid irreversible deterministic effects to the patient, such as tissue necrosis.

The next morning, a WB acquisition was performed at 7:40 $\left(T_{20 h}\right)$, followed by a SPECT/CT acquisition. A last imaging session was realized at 14:04 $\left(\mathrm{T}_{26 \mathrm{~h}}\right)$ with WB and SPECT/CT images to refine the first dose estimation made on the day of the incident. Imaging times and types are synthesized on Figure 3.

\subsection{Volume estimation}

After extravasation, the ${ }^{177}$ Lu-DOTATATE spread heterogeneously in the subcutaneous tissue. Due to very low contrast, CT could not be used to determine the extravasation volume. For the initial dose estimation, the extravasation volume was determined using a 3D threshold on a ${ }^{\circledR}$ Syngo Via software (Siemens).

For the second dose estimation, we tried to get a better idea of the dose distribution by defining 3 volumes of interest using 3D threshold on SPECT images: one large volume encompassing the extravasation area and including low uptake voxels, one medium volume close to the volume used for the initial dose estimation and one smaller volume corresponding to the voxels with higher uptake. This approach was used to get a range of dose estimations, more informative than one single value.

\subsection{Dose calculation}

The MIRD formalism was used to determine the dose in the extravasated area (9). Given the low energy of beta emissions from ${ }^{177} \mathrm{Lu}$ and the high uptake of the extravasation region, only self-irradiation was considered for dose calculation.

For the first dose estimation, the percentage of activity in the arm related to the activity in the whole body was used to estimate the activity in the extravasated area. This calculation was made on the geometrical mean on anterior and posterior WB $\mathrm{T}_{2 \mathrm{~h}}$ images. WB images were processed with ImageJ v1.51n (10) and SPECT/CT images were processed on Syngo ${ }^{\circledR}$ software (Siemens).

$T_{5 h}$ SPECT/CT images were used to determine the volume of the extravasated area. Effective period was determined on ROI drawn around the arm on the geometrical mean of $T_{2 h}$ and $T_{5 h} W B$ images.

For the second more accurate dose estimation taking all images into account, activity in the extravasated area was determined using the calibration factor estimated on phantoms data for SPECT images. VOI were defined on SPECT images.

As no SPECT/CT images were acquired at $\mathrm{T}_{2 \mathrm{~h}}$. To get an estimation of the activity at this time point, ROI around the extravasation were drawn on geometric mean of whole body images acquired at $T_{2 h}$ and $T_{5 h}$. 
A ratio between counts in $\mathrm{ROI}$ at $\mathrm{T}_{2 \mathrm{~h}}$ and $\mathrm{T}_{5 \mathrm{~h}}$ was calculated and applied to activity estimated on SPECT CT at $T_{5 h}$ to approximate the activity at $\mathrm{T} 2 \mathrm{~h}$, as shown in equation 1.

$$
A_{\text {SPECT }_{T 2 h^{*}}}=A_{S P E C T_{T 5 h}} \frac{N b \text { counts ROI } \operatorname{arm}_{W B_{T 2 h}}}{N b \text { counts ROI } \operatorname{arm}_{W B_{T 5 h}}}
$$

Effective period was determined with activity estimated on SPECT images and $\mathrm{A}_{\text {SPECT T2h*. }}$

For both dose estimations, cumulated activity was determined as the area under the curve of the mono exponential fit of activity calculated on images.

For dose calculation, we used the approach detailed by Sandström et al for kidney ${ }^{177}$ Lu-DOTATATE dosimetry based on a dose factor (DF), which is the absorbed energy per time-integrated activity concentration in nGy.kg/(MBq.s) (8). DF factors calculation are based on Radar website data (11). DF values are between 23.9 and $24.8 \mathrm{nGy} . \mathrm{kg} /(\mathrm{MBq} . \mathrm{s})$ for spheres between $100 \mathrm{~g}$ and $2000 \mathrm{~g}$. We chose to use a unique approximate value of 24 nGy.kg/(MBq.s) for dose estimations.

\section{Results}

\subsection{Phantom study}

The relationship between the counting rate in SPECT images and activity in the cylinder is well fit using a linear curve (Figure 4, left). The slope of the straight line gives a calibration coefficient of 11.55 counts/s/MBq.

Activity in the verification phantom was estimated using this calibration factor and counts in VOI determined on CT images (Figure 4). The average error between estimated activity in the images and activity in the cylinder measured with dose calibrator is $-1.27 \%$ ( $\min -4.30 \%, \max 1.98 \%$ ).

\subsection{Patient preliminary dose estimation}

The residual activity in the vial after infusion measured with a calibrated dose meter was $226 \mathrm{MBq}$. Thus, the total activity delivered to the patient was $7569 \mathrm{MBq}$ at the time of injection. A preliminary dose estimation was performed using whole body image at $\mathrm{T}_{2 \mathrm{~h}}$ and $\mathrm{T}_{5 \mathrm{~h}}$ (Figure 5), and SPECT/CT images at $\mathrm{T}_{5 \mathrm{~h}}$ (Figure 6), to determine whether a surgical rinse of the arm was necessary.

Unfortunately, the extravasated area was not entirely in the field of view of the first acquisition (Figure 5, left). The number of counts outside the field of the first image was roughly estimated using $T_{5 h} W_{B}$ image. This image was truncated to get a similar truncation as $T_{2 h}$ WB image, and the ratio of counts in the arm area in the truncated and in the whole image was calculated. The count loss due to truncation in the first image was estimated to $5 \%$ and accounted for in the count calculation. 
Using the first whole body image and correcting for truncation, $74 \%$ of the injected ${ }^{177}$ Lu activity was concentrated in the upper part of the arm compared to the whole body. A monoexponential curve was fitted based on the counts measured on the arm and whole body counts at $T_{2 h}$ and $T_{5 h}$. The effective period was around $1.90 \mathrm{~h}$ in the arm and $4.36 \mathrm{~h}$ in the whole body. Given the risk of necrosis for the patient and the lack of reliability of a monoexponentiel fit on 2 points, we chose to use the largest of these computed effective periods for evaluating the dose in the arm, namely the whole body period.

An extravasated volume of around $400 \mathrm{~mL}$ was estimated based on $\mathrm{T}_{5 \mathrm{~h}}$ SPECT/CT image. The evaluated dose was around $8 \mathrm{~Gy}$. This value, under the order of magnitude of necrosis risk dose for interstitial tissue in extravasation case (around $20 \mathrm{~Gy}$ ) (12) eliminated the need for a surgical rinse of the arm of the patient. Nevertheless, adapted interventions were applied to the patient: the nurses warmed and elevated his arm during the afternoon and the evening after the extravasation. The patient performed repeated self-massages of the upper part of the arm in the evening and in the night.

\subsection{Accurate estimation of activity from SPECT/CT images after extravasation}

Whole body (Figure 7) and SPECT/CT images acquired the day after extravasation allowed a more precise evaluation of the dose in the arm and show a reduction of the extravasation in the arm.

The effective period calculated using counts on $T_{2 h}, T_{5 h}, T_{20 h}$ and $T_{26 h}$ was $3.5 \mathrm{~h}$ in the arm and $10.5 \mathrm{~h}$ in the whole body.

The volumes of the 3 threshold-based $\mathrm{VOI} \mathrm{V}_{1}, \mathrm{~V}_{2}$ and $\mathrm{V}_{3}$ were respectively $1000 \mathrm{~mL}, 400 \mathrm{~mL}$ and $180 \mathrm{~mL}$. These VOI are shown on the $T_{5 h}$ SPECT/CT (Figure 8). These VOI were drawn on the $T_{5 h}$ SPECT/CT and reported on the $\mathrm{T}_{20 \mathrm{~h}}$ and $\mathrm{T}_{26 \mathrm{~h}}$ SPECT images.

Time activity curves in the extravasation area for the $3 \mathrm{VOI}$ are plotted in Figure 9. Monoexponential curves following Eq.2 model were fitted to the data to estimate effective period with parameters shown in Table 4, where $A(t)$ is the activity in the arm, $A_{0}$ is the estimated activity at $T_{0}$ and $T_{\text {eff }}$ the effective period.

$$
A(t)=A_{0} e^{-\ln (2) t / T_{e f f}}
$$

$A_{0}$ is a purely theorical value, as $T_{0}$ corresponds to the beginning of the infusion. The infusion process is slow and the activity cannot be instantaneously spread in the extravasated area. It nevertheless gives an idea of the amount of activity considered in each of the 3 dose estimations. 


\begin{tabular}{|llll|}
\hline & Effective period $\mathrm{T}_{\text {eff }}(\mathrm{h})$ & $\mathrm{A}_{0}(\mathrm{MBq})$ & Estimated dose (Gy) \\
\hline $\mathrm{V}_{1}$ & 3.36 & 5254 & 2.21 \\
$\mathrm{~V}_{2}$ & 3.14 & 4110 & 4.09 \\
$\mathrm{~V}_{3}$ & 2.84 & 3904 & 7.81 \\
\hline
\end{tabular}

Table 4. Estimation of effective period, activity at infusion time and corresponding local absorbed dose for volumes $V_{1}, V_{2}$ and $V_{3}$

Based on these models, and using the mean dose coefficient of $24 \mathrm{nGy} \cdot \mathrm{kg} /(\mathrm{MBq} . \mathrm{s})$, estimated local dose ranged from 2.21 to $7.81 \mathrm{~Gy}$, according to the volume considered (Table 4).

\section{Discussion}

\subsection{Effects of extravasation}

The range of dose leading to deterministic effects depends on the type of radiation and the exposed organ. For skin, it is well reported for external exposure, such as fluoroscopy (13). For extravasation cases, a few studies linked dose values to observed effects. Shapiro et al reported a threshold dose of 20 Gy to observe radiation induced injury in for extravasation of radiopharmaceutical in interstitial tissue (12). Bonta et al. reported skin injury and desquamation with a dose between 22 and 36 Gy during an extravasation of 1311-Metaiodobenzylguanidine (14). Williams et al reported wet desquamation with a dose between 20 and 40 Gy during an extravasation of Yttrium-90-Ibritumomab Tiuxetan (15).

Our estimation of absorbed dose in the subcutaneous tissue in the arm of the patient is comprised between 2 and $8 \mathrm{~Gy}$. We can hypothesize that local absorbed skin dose (especially hypodermic dose) is similar to subcutaneous tissue dose (Figure 8). ICRP reported a threshold between 3 and 6 Gy for deterministic effects for the skin, this value corresponds to the dose level where $1 \%$ of exposed persons would experience the effect (16).

The patient was seen the day after, 6 days, and 3 weeks after the incident. He did not show any clinical sign of irradiation, like erythema, and did not report any pain in the area of extravasation. Though large, the range of doses that our model hypothesized was therefore accurate enough to estimate the risk.

\subsection{Comparison of dose estimations}

We performed two dose estimations: a first estimation in the evening and another more accurate estimation in the days following the incident. The first dose evaluation was necessary to rapidly evaluate the need of a surgical intervention. The purpose of the second one was to get a more accurate result to confront with the patient follow-up. Dosimetric data for extravasation cases are scarce in the literature 
(1). No data have been published for ${ }^{177}$ Lu-DOTATATE, it was then really difficult to estimate the consequences of the extravasation without dosimetric evaluation.

Both estimations cannot strictly be compared, as the effective period was not evaluated on the same number of points. Nevertheless, we can compare the activity ratio between the arm and the whole body estimated from the whole body images and SPECT images. We estimated than $74 \%$ of activity was in the arm with $T_{2 h}$ whole body images. Activity estimated using $V_{1}$ was estimated to $5254 \mathrm{MBq}$ at the time of injection, which represents $69 \%$ of injected activity. Given the uncertainty of both calculation methods, we can consider these 2 results as concordant.

The second activity estimation relied on the calibration factor determined on the SPECT phantom study. A different approach to estimate the activity or even verify our calculations could have been to make the assumption that the total activity of the patient was in the first whole body image counts and use a ratio between the counts in the arm and in the whole body with an estimation of the volume of interest made on SPECT/CT images. Unfortunately, the patient urinated before its first whole body acquisition. With no knowledge of the activity in the first miction, we could not base our estimation on this whole body image based approach. Retrospectively, the percentages of activity in the arm calculated using the whole body images $\left(74 \%\right.$ at $\left.\mathrm{T}_{2 \mathrm{~h}}\right)$ and SPECT images $\left(69 \%\right.$ at $\left.\mathrm{T}_{0}\right)$ are very close. If we hypothesized that the first miction activity was negligible and that the first whole body image represents the total activity administered to the patient, the error on the dose estimation would have been very low, compared to the overall dose uncertainty.

\subsection{Distribution of ${ }^{177}$ Lu-DOTATATE after extravasation}

Whole body images show the efficiency of adapted interventions and lymphatic drainage, reducing extravasation in patient, with a rather short effective period, of around 3 hours.

Specific interventions were applied to the patient, which were detailed in our local procedure for extravasation cases. After the incident, we completed this document with the acquisitions of WB and SPECT/CT images as quickly as possible after the detection of the incident. Images should be acquired at least twice a day, as it has been done for this patient.

The distribution of ${ }^{177}$ Lu-DOTATATE in the patient the day after the injection (Figure 7) shows a classical pattern of distribution for GEP NET patients treated with ${ }^{177}$ Lu-DOTATATE. After lymphatic drainage, the product distributed in blood flow and accumulated in tumors and metastases of the patient. The impact of extravasation on the efficiency of the treatment is hard to evaluate but most of the product in all likelihood reached its target. Furthermore, after having completed the treatment (with administration of 3 subsequent cycles of Lutathera), the patient reported a significant improvement of carcinoid syndrome with disparition of flush, and diminution of diarrhea. Tumoral disease remained stable 18 months after the end of PRRT.

\subsection{Limits of the dose estimation}


The dose estimation resulting from the extravasation has several limits. The determination of the volume used for dose calculation was challenging, due to its complex shape and its decrease over time. We therefore chose 3 volumes from $480 \mathrm{~mL}$ to $1 \mathrm{~L}$, to get a plausible range of absorbed dose and an idea of the dose distribution in the extravasated area. A voxel-based approach for dose determination, which generates dose-volume histograms, could have been useful in this case. Unfortunately, a voxel-based dosimetry software is not yet routinely available in the center.

Several corrections have not been applied to the images: the dead-time effect has not been taken into account, and counts from the arm VOI were not corrected for partial volume effect. Data were not available in the center to estimate the impact of these two effects on the quantification of ${ }^{177}$ Lu SPECT images. Moreover, the complex shape of the extravasated volume made a robust partial volume effect correction very tedious. A recent study by Uribe et al. evaluated the deadtime for ${ }^{177} \mathrm{Lu}$ on a Symbia system (17) and reported a 10\% count loss for images acquired 3 hours after injection for patients treated with 7.4 GBq of ${ }^{177} \mathrm{Lu}$, and no count loss for images acquired later. These data have been measured on a $3 / 8$ " crystal camera and cannot be used to correct for deadtime on our data. Nevertheless, taking deadtime into account would have led to a slightly increased activity estimation on $T_{2 h}$ images, and a slightly decreased effective period. The resulting impact on dose estimation would be a few percent decrease.

Despite these limitations, the dose estimation is coherent with the absence of observable effects.

\section{Conclusion}

Local dose was estimated for an extravasation case in the arm of a patient during a ${ }^{177}$ Lu-DOTATATE infusion, using SPECT/CT, WB images and a quantitative procedure, initially developed for renal dosimetry. The estimated dose in the arm, comprised between 2 and $8 \mathrm{~Gy}$ based on the VOI considered to define the extravasated volume, is in line with the absence of detectable effects. Adapted interventions promoted the elimination of ${ }^{177}$ Lu-DOTATATE in the arm and limited the absorbed dose in non-target tissues.

\section{Declarations}

\section{Ethics approval and consent to participate}

Ethical approval was waived by the local Ethics Committee of Hospices Civils de Lyon in view of the retrospective nature of the study and all the procedures being performed were part of the routine care.

\section{Consent for publication}

Patient signed informed consent regarding publishing his data and images.

\section{Availability of data and material}


The datasets generated and analysed during the current study are available from the corresponding author on reasonable request.

\section{Competing interests}

No financial or non-financial competing interest

\section{Funding}

Not applicable

\section{Authors' contributions}

Perrine Tylski's contributions were: collecting patient and phantom data, analyzing phantom and patient data, writing the manuscript

Géradine Pina Jomir's contributions were: following the patient, analyzing patient's data and writing the manuscript

Claire Bournaud's contributions were: following the patient and writing the manucript

Patrice Jalade's contributions were: analyzing phantom and patient data and writing the manuscipt

\section{Acknowledgements}

Not applicable

\section{References}

1. van der Pol J, Vöö S, Bucerius J, Mottaghy FM. Consequences of radiopharmaceutical extravasation and therapeutic interventions: a systematic review. Eur J Nucl Med Mol Imaging. 2017;44:1234-43.

2. Strosberg JR, Wolin EM, Chasen B, et al. NETTER-1 phase III: Progression-free survival, radiographic response, and preliminary overall survival results in patients with midgut neuroendocrine tumors treated with 177-Lu-dotatate. 2016.

3. Ljungberg M, Celler A, Konijnenberg MW, Eckerman KF, Dewaraja YK, Sjögreen-Gleisner K. MIRD pamphlet no. 26: joint EANM/MIRD guidelines for quantitative 177Lu SPECT applied for dosimetry of radiopharmaceutical therapy. Journal of nuclear medicine. 2016;57:151-62.

4. Zaknun JJ, Bodei L, Mueller-Brand J, et al. The joint IAEA, EANM, and SNMMI practical guidance on peptide receptor radionuclide therapy (PRRNT) in neuroendocrine tumours. Eur J Nucl Med Mol Imaging. 2013;40:800-16.

5. Schlenkhoff CD, Essler M, Ahmadzadehfar H. Possible Treatment Approach to an Extravasation of 177Lu-PSMA-617. Clinical nuclear medicine. 2017;42:639-40. 
6. Jüptner M, Zuhayra M, Assam I, Lützen U. Successful handling of an accidental extravasation of 177Lu-PSMA-617 in the treatment of advanced prostate cancer. Nuklearmedizin. 2018;57:N10-2.

7. Cheong B, Muthupillai R, Rubin MF, Flamm SD. Normal values for renal length and volume as measured by magnetic resonance imaging. Clinical journal of the American Society of Nephrology. 2007;2:38-45.

8. Sandström M, Garske U, Granberg D, Sundin A, Lundqvist H. Individualized dosimetry in patients undergoing therapy with 177 Lu-DOTA-D-Phe 1-Tyr 3-octreotate. Eur J Nucl Med Mol Imaging. 2010;37:212-25.

9. Bolch WE, Eckerman KF, Sgouros G, Thomas SR. MIRD pamphlet no. 21: a generalized schema for radiopharmaceutical dosimetry-standardization of nomenclature. J Nucl Med. 2009;50:477-84.

10. Rueden CT, Schindelin J, Hiner MC, et al. ImageJ2: ImageJ for the next generation of scientific image data. BMC Bioinform. 2017;18:529.

11. Stabin M, Siegel J, Hunt J, Sparks R, Lipsztein J, Eckerman K. RADAR: the radiation dose assessment resource-an online source of dose information for nuclear medicine and occupational radiation safety. J Nucl Med. 2001;42:243P.

12. Shapiro B, Pillay M, Cox P. Dosimetric consequences of interstitial extravasation following iv administration of a radiopharmaceutical. Eur J Nucl Med. 1987;12:522-3.

13. Balter S, Hopewell JW, Miller DL, Wagner LK, Zelefsky MJ. Fluoroscopically guided interventional procedures: a review of radiation effects on patients' skin and hair. Radiology. 2010;254:326-41.

14. Bonta DV, Halkar RK, Alazraki N. Extravasation of a therapeutic dose of 1311metaiodobenzylguanidine: prevention, dosimetry, and mitigation. J Nucl Med. 2011;52:1418-22.

15. Williams G, Palmer MR, Parker JA, Joyce R. Extravazation of therapeutic Yttrium-90-Ibritumomab tiuxetan (Zevalin ${ }^{\circledR}$ ): A case report. Cancer Biother Radiopharm. 2006;21:101-5.

16. ICRP 2007. The 2007 Recommendations of the International Commission on Radiological Protection. ICRP Publication 103. 37 (2-4).

17. Uribe CF, Esquinas PL, Gonzalez M, Zhao W, Tanguay J, Celler A. Deadtime effects in quantification of 177 Lu activity for radionuclide therapy. EJNMMI physics. 2018;5:2.

\section{Figures}



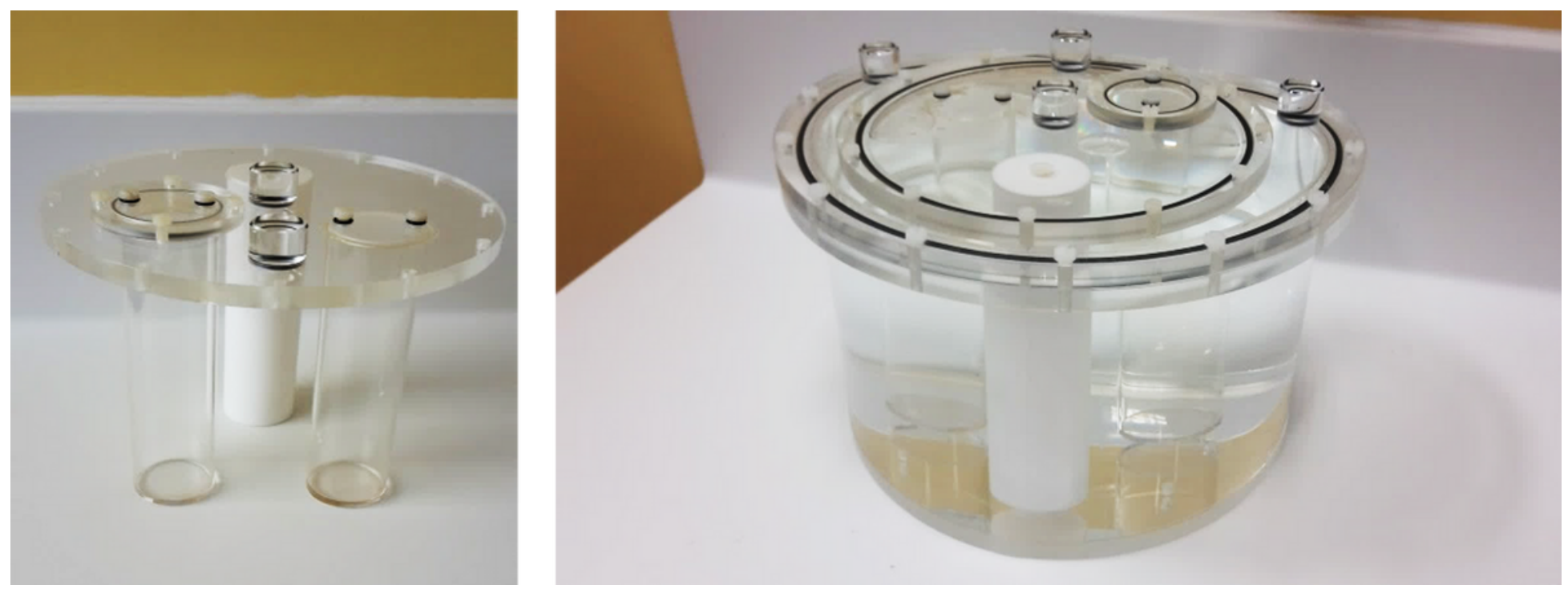

\section{Figure 1}

3-cylinder cover (left), and calibration phantom with body phantom and 3-cylinder cover (right)
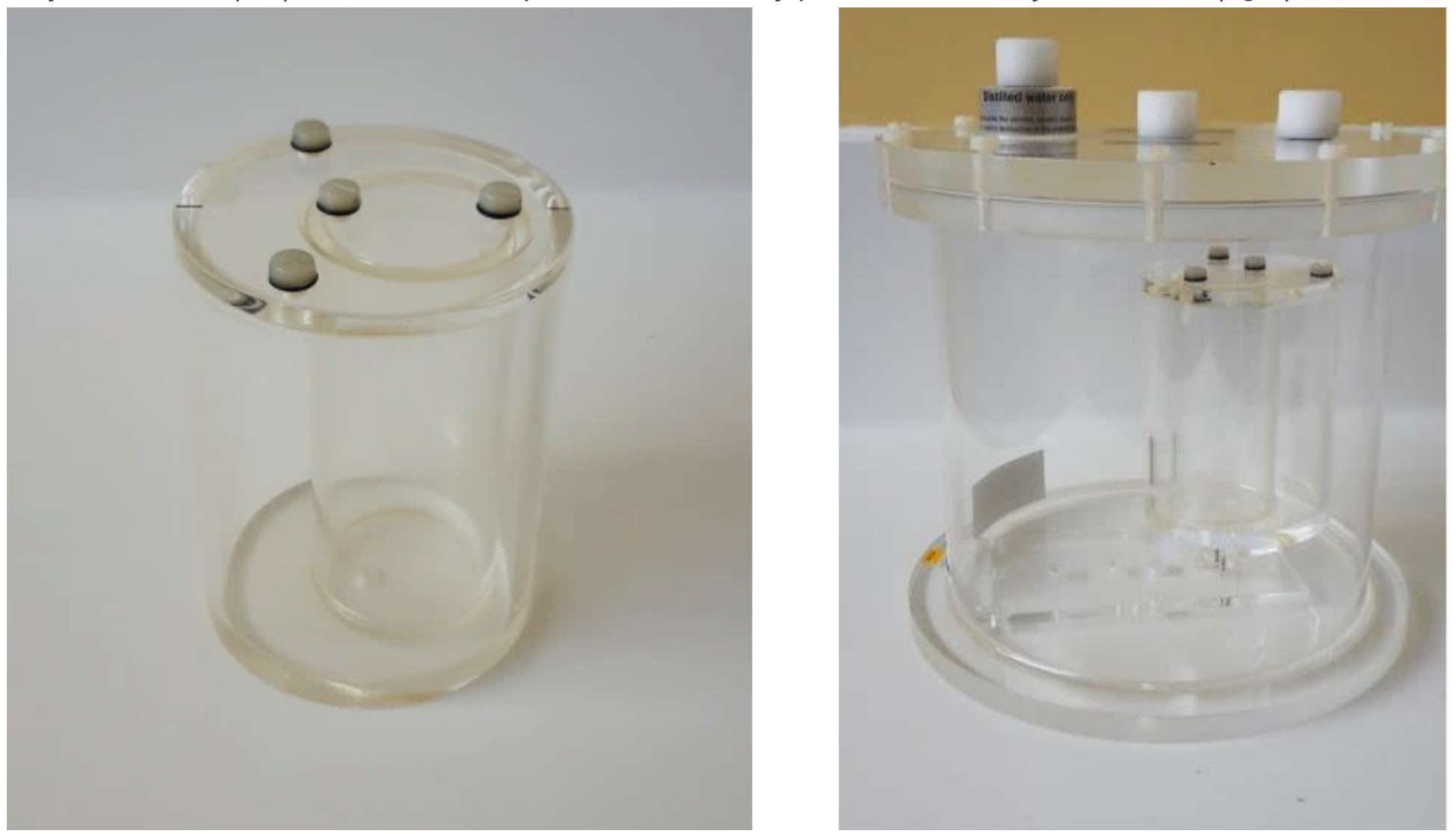

Figure 2

Heart phantom insert (left), and an example of a verification phantom setup with the heart insert placed in a head phantom (right) 


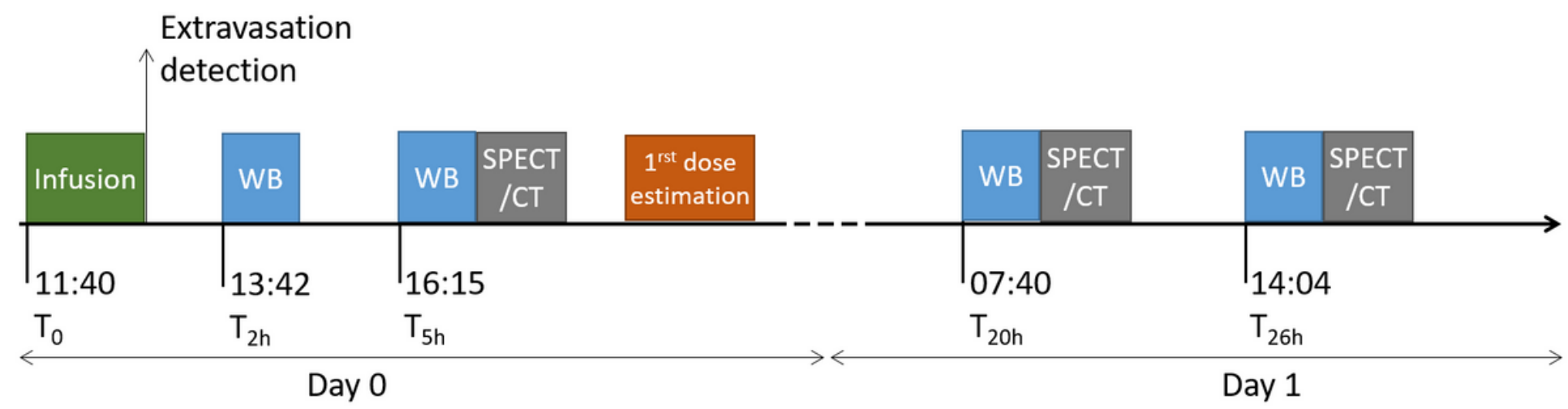

Figure 3

Chronology of image acquisitions from the beginning of infusion
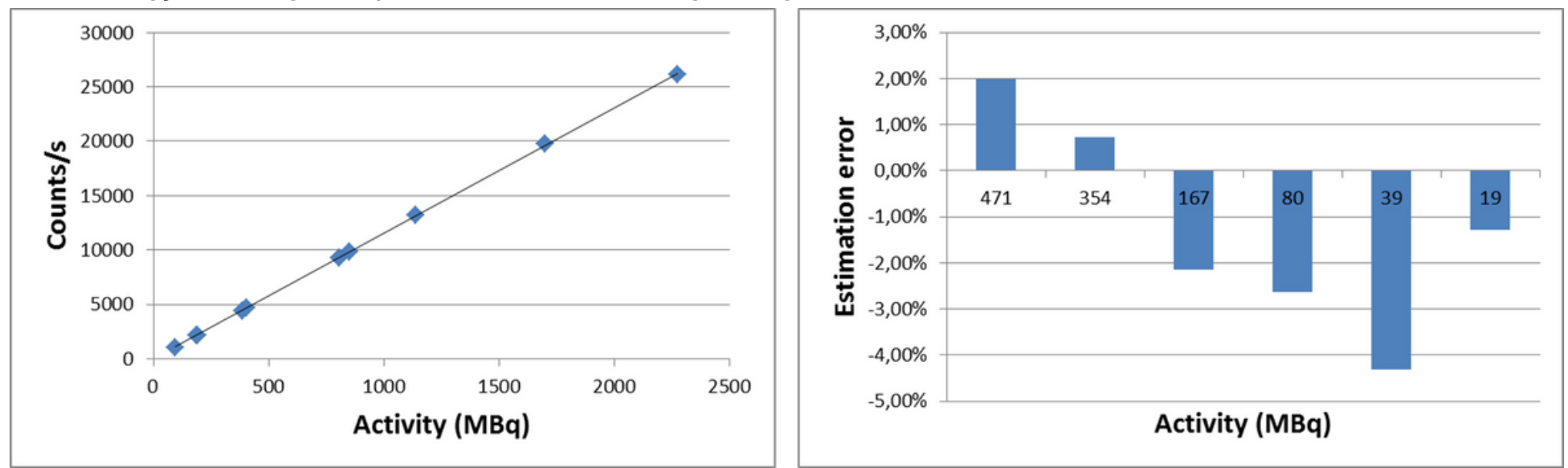

Figure 4

Counting rate versus activity for the calibration step (left) and error in activity estimation for the verification step (right) 

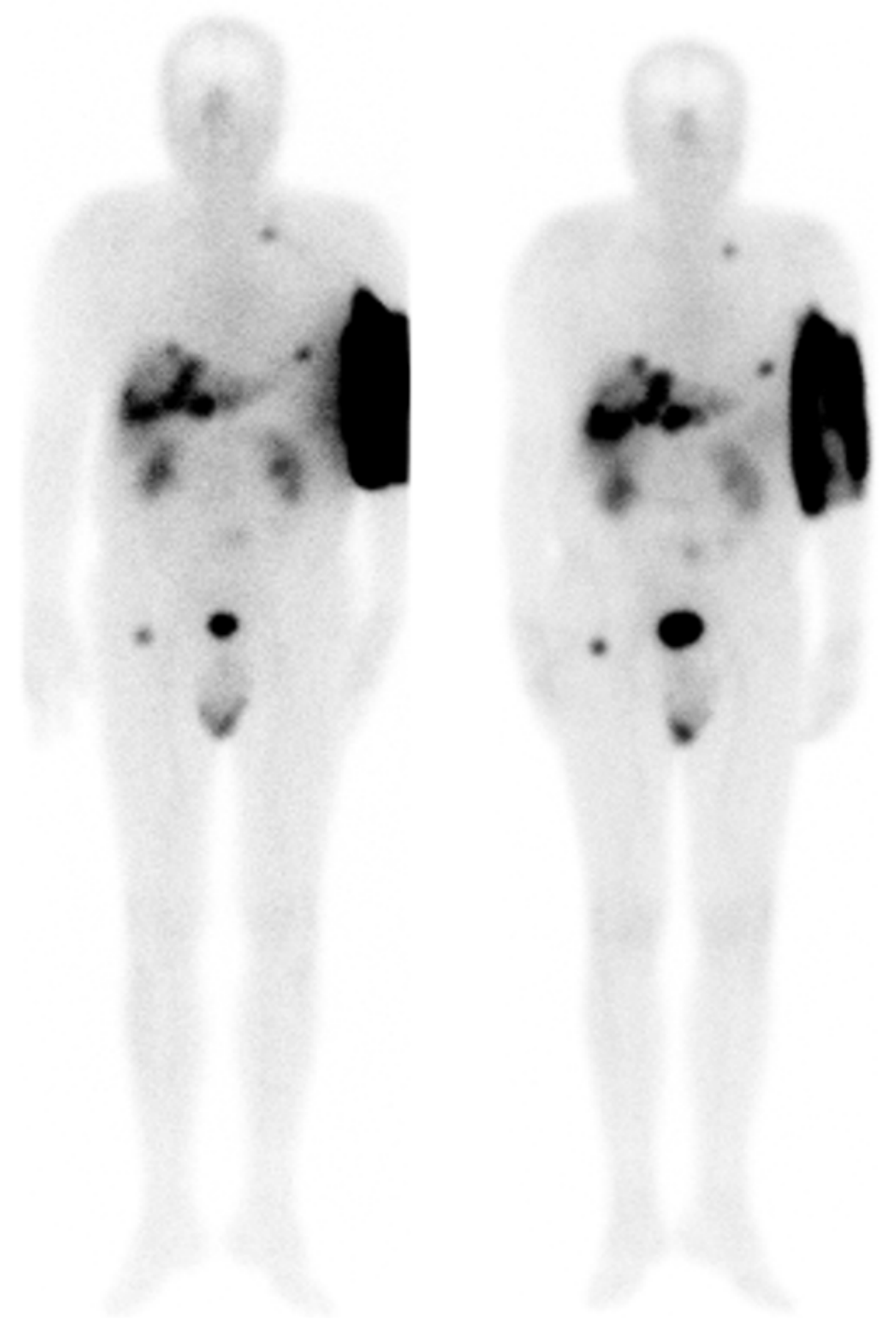

Figure 5

Geometric mean of whole body images acquired at T2h (left) and T5h (right) 

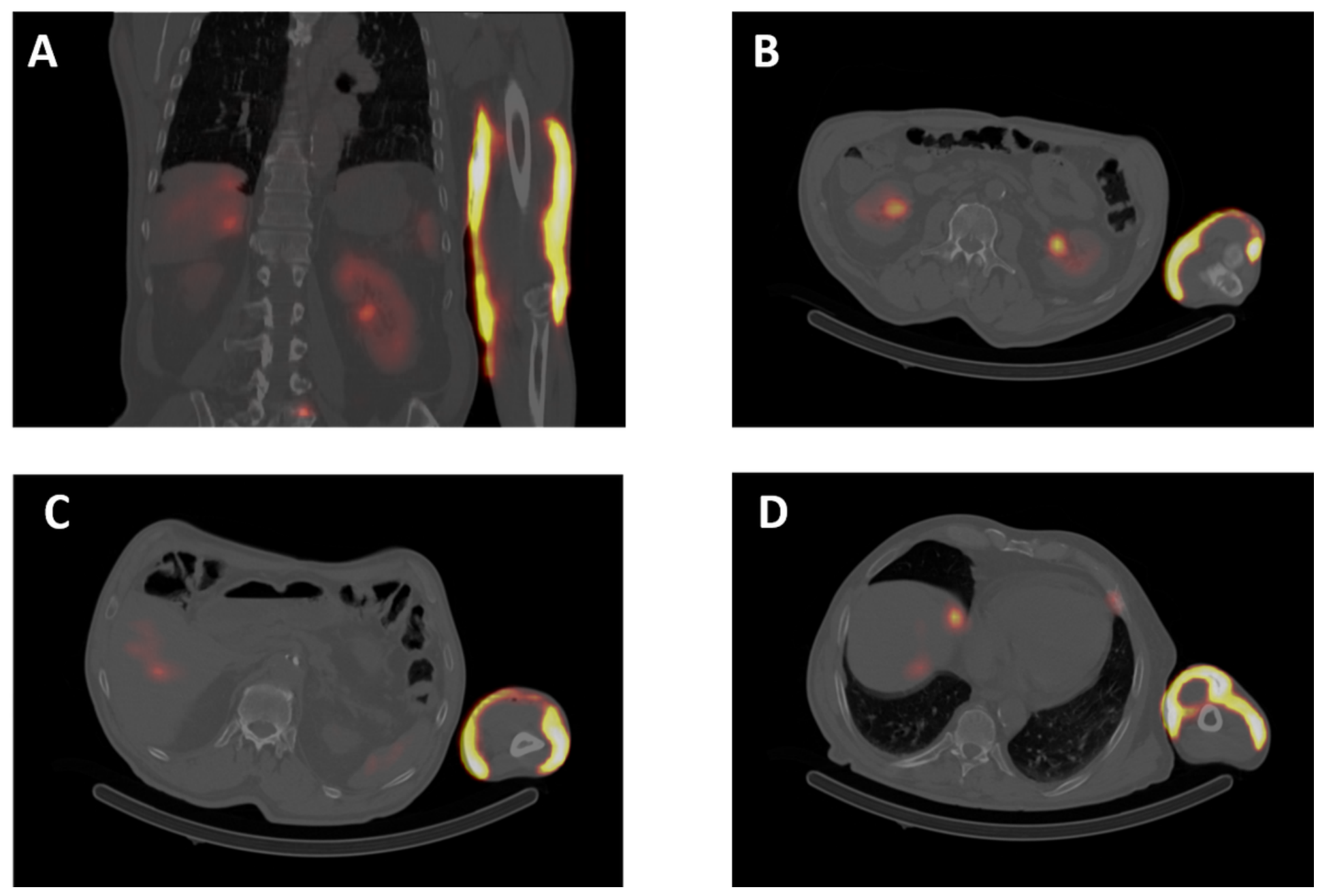

Figure 6

Coronal slice (A) and axial slices (B,C,D) of T5h SPECT/CT showing the subcutaneous distribution of the extravasation in the arm 

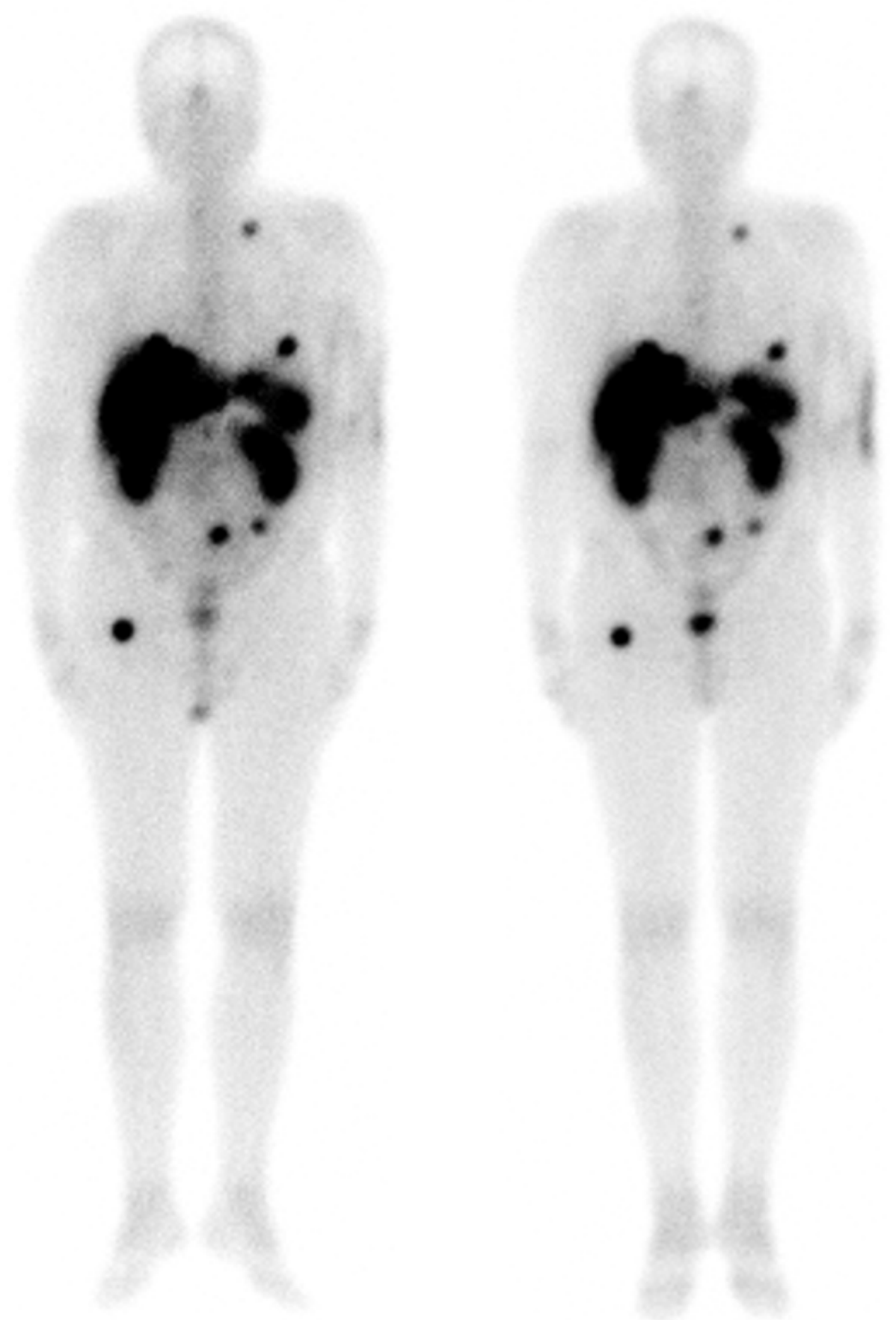

Figure 7

Geometric mean of whole body images acquired at T20h (left) and T26h (right) 

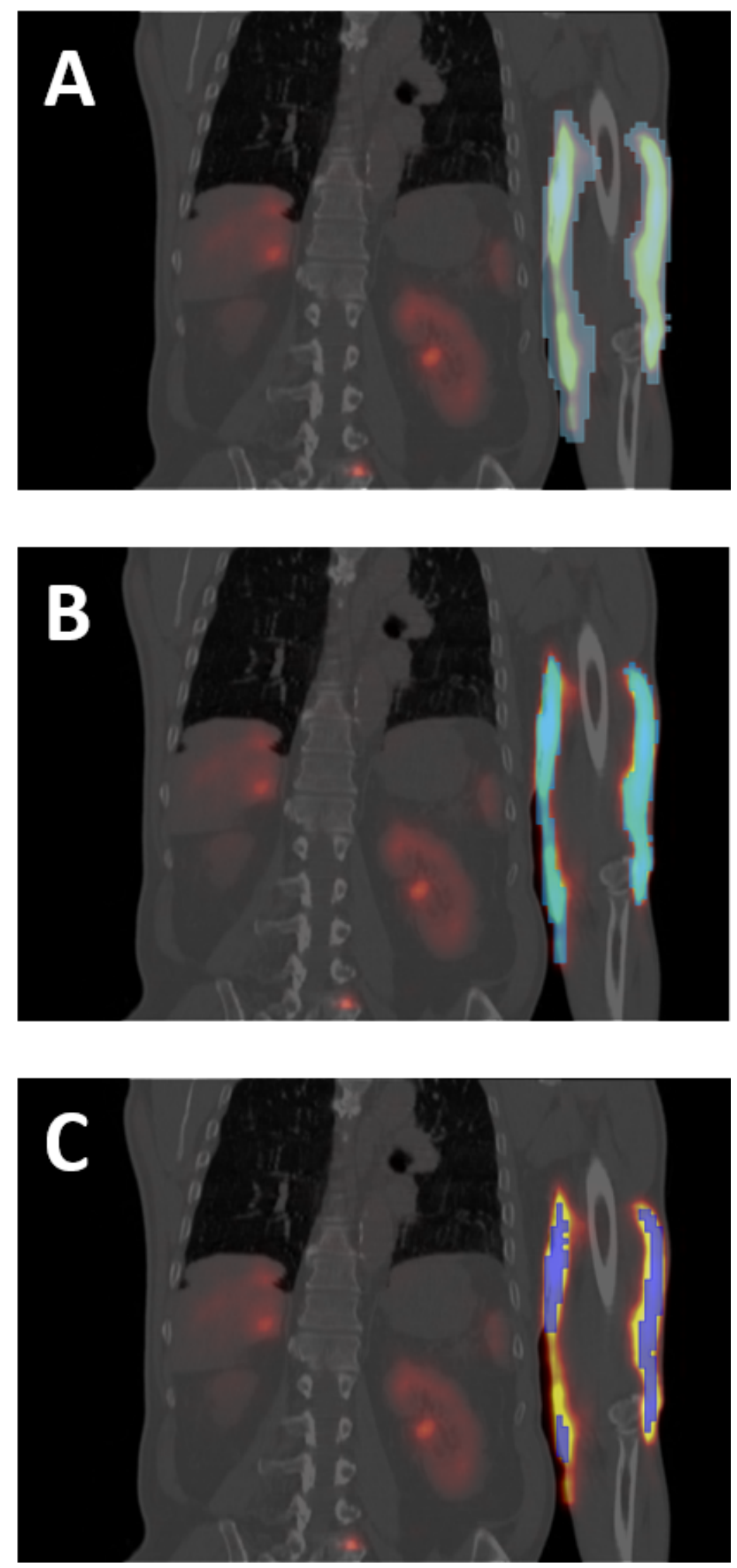

Figure 8

Coronal slice of T5h SPECT/CT with superimposed VOI corresponding to $1000 \mathrm{~mL}$ (A), $400 \mathrm{~mL}$ (B) and $180 \mathrm{~mL}(\mathrm{C}) \mathrm{VOls}$ 


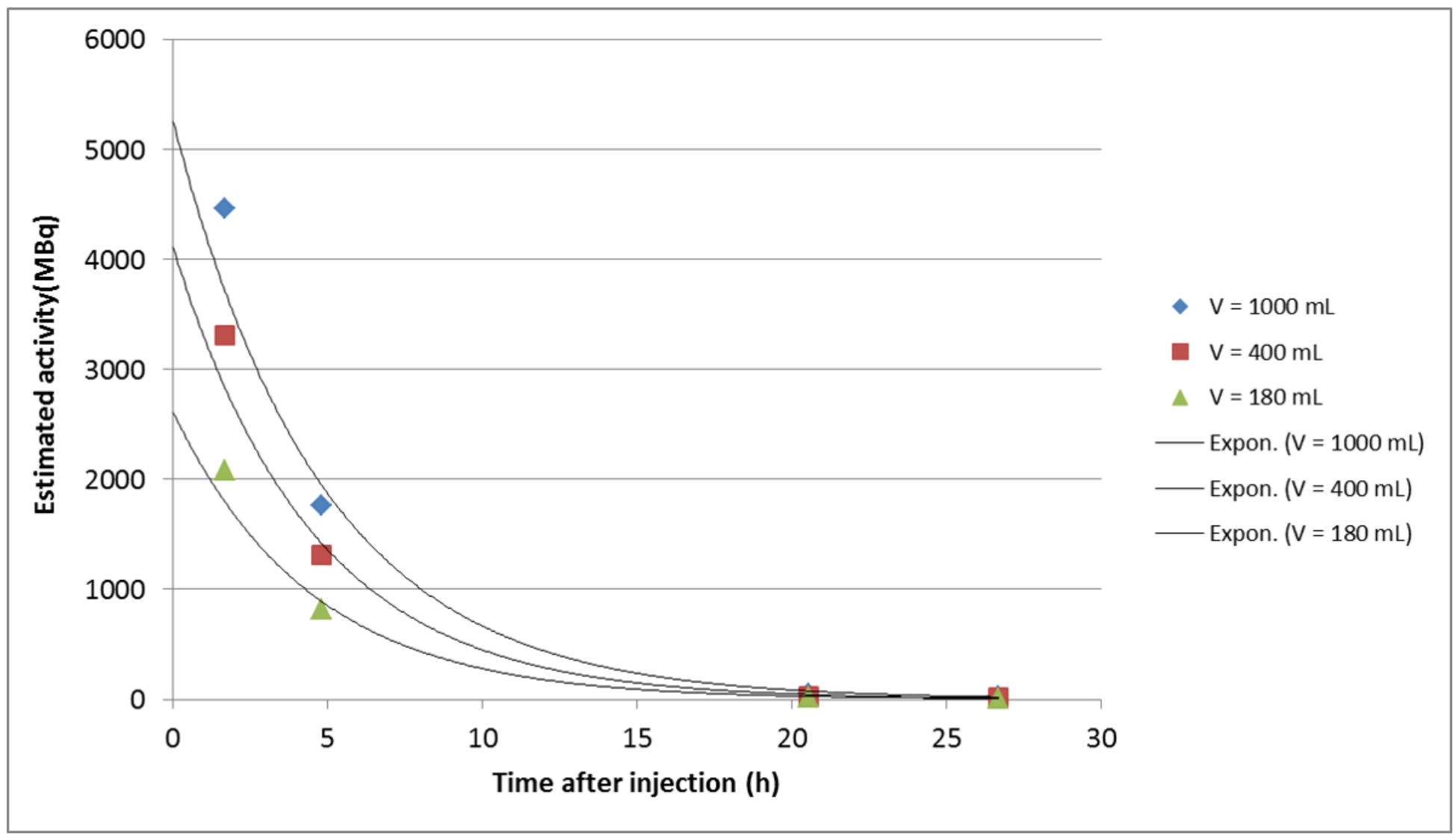

Figure 9

Estimated time activity curves for the $3 \mathrm{VOI}$ of 1000,400 and $180 \mathrm{~mL}$ 